\title{
InP microdisk lasers integrated on Si for Optical Interconnects
}

\author{
Geert Morthier, Senior Member, IEEE, Thijs Spuesens, Member IEEE, Pauline Mechet, Gunther \\ Roelkens, Senior Member, IEEE and Dries Van Thourhout, Senior Member, IEEE
}

\begin{abstract}
We review recent theoretical and experimental work on InP membrane microdisk lasers heterogeneously integrated on SOI and coupled to a Si bus waveguide. The lasers can now be fabricated with very high yield, have typical threshold currents of $0.5 \mathrm{~mA}$ and output powers of tens of $\mu \mathrm{W}$, while the total power consumption is restricted to $5 \mathrm{~mW}$. First, we describe various improvements in the fabrication technology and interesting results on the uniformity in device characteristics. In a second part, unidirectional behaviour and reflection sensitivity are briefly discussed. The third part is focused on optical signal regeneration with microdisk lasers. The last part contains a brief summary of optical interconnects based on heterogeneously integrated microdisk lasers and heterogeneously integrated photodetectors.
\end{abstract}

Index Terms-silicon photonics, microdisk lasers, optical interconnect, heterogeneous integration

\section{INTRODUCTION}

$\mathrm{H}$ eterogeneously integrated microdisk lasers have been studied since a decade [1] and were among the first electrically pumped InP lasers integrated on silicon. Because of the high optical confinement in the InP membranes, the small disk diameters $(10 \mu \mathrm{m}$ or lower) and the weak, evanescent coupling to the underlying Si bus waveguide, such microdisk lasers can have relatively low threshold gain and threshold current and are therefore rather well suited as sources for optical interconnects [2,3]. This is especially relevant as silicon photonics is considered more and more as the platform in which to implement optical interconnects [4].

While photonic crystal lasers are known to exhibit lower thresholds, the difficulty in pumping them electrically and their very weak output powers make them so far less suitable for such applications, unless transmission losses are extremely low [5]. Off-chip light sources on the other hand are more difficult to use with complex interconnect networks [6].

Heterogeneously integrated microdisk lasers (Figure 1) make use of the high optical confinement that can be obtained in a thin $(0.6 \mu \mathrm{m})$ bonded InP membrane. A centrally located top contact on this thin membrane furthermore allows a whispering gallery mode with low loss, while giving high loss for all other modes. Heterogeneously integrated microdisk lasers are relatively easy to incorporate in silicon photonic integrated circuits. Indeed, ring or disk lasers don't require reflecting facets or diffraction gratings

Manuscript received July 22, 2014. This work was supported in part by the European FP7 project HISTORIC.

G. Morthier, T. Spuesens, P. Mechet, G. Roelkens, D. Van Thourhout are with the Photonics Research Group, Department of Information Technology, Ghent University - imec, B-9000 Gent, Belgium. fax: +32 9 2643593; e-mail: morthier@ intec.ugent.be. and are relatively easy to fabricate. As will be discussed further on, they can be configured to lase in a predefined unidirectional mode and to be less sensitive to external reflections [7-9].

The typical threshold current of our microdisk lasers is around $0.5 \mathrm{~mA}$, with an output power at $5 \mathrm{~mA}$ up to a few hundreds of $\mu \mathrm{W}$ (in the silicon waveguide) and total power consumption of a few $\mathrm{mW}$ [10]. The free spectral range of the microdisk resonator for a diameter of $7.5 \mu \mathrm{m}$ is slightly over $30 \mathrm{~nm}$, giving the lasers a stable single mode behavior with a side mode suppression over $40 \mathrm{~dB}$. For a $7.5 \mu \mathrm{m}$ diameter $\mathrm{CW}$-operated microdisk, a minimum threshold current of $0.22 \mathrm{~mA}$ and a maximum single-sided slope efficiency of $57 \mu \mathrm{W} / \mathrm{mA}$ have been observed [11].

Microdisk and ring lasers have also been studied extensively in the past decade, because of their suitability as bistable lasers for optical switching or signal processing applications such as all-optical flip-flops, gates and wavelength converters [10,12-16].

Several improvements in the fabrication technology have been successfully implemented in recent years. This has led to bonding yields of nearly $100 \%$ [17] and very good uniformity in the lasing characteristics of microdisk lasers fabricated on the same SOI die [18]. In addition to the bonding, there are a number of other aspects which require special attention though (Figure 1):

- Overlap of the top contact with the whispering gallery mode must be avoided, as this leads to excess loss with the very thin $(0.5 \mu \mathrm{m})$ membranes used.

- Carefull alignment of the microdisks with the underlying silicon wire waveguide to obtain optimum coupling

- The small volume of the membrane and its adhesive bonding onto SOI give rather poor heat sinking.

Fabrication and design have been optimized to account for these potential problems and this is explained in more detail in the first part of this paper.

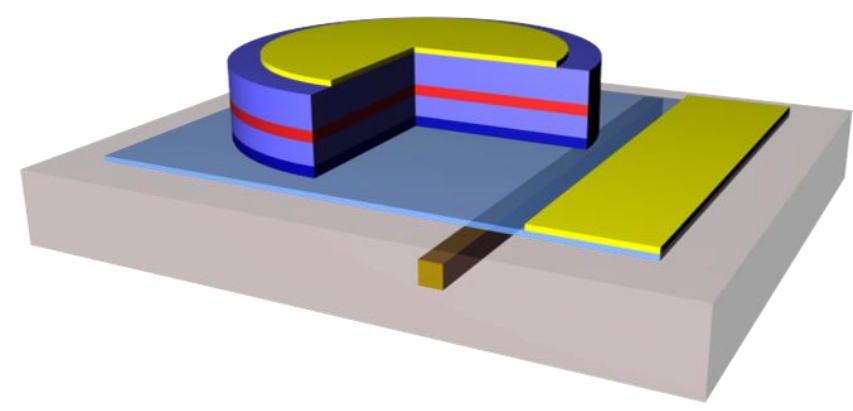


Figure 1. Schematic view of an InP microdisk laser heterogeneously integrated onto SOI.

For optical interconnect even more than for long distance communications, high efficiency and isolator-free operation of light sources are of paramount importance. Some theoretical and experimental work has therefore been dedicated to unidirectional operation (in a predefined direction) and to the feedback sensitivity of the microdisk lasers and it is the subject of the second part of this paper.

Asymmetrically terminated ring or disk lasers, which are necessary for unidirectional operation, also lend themselves very well for low-power optical signal regeneration. Integration of such a microdisk laser with a photodiode could actually serve as a regenerative receiver with low power consumption. We will briefly summarize the results obtained so far on this $2 \mathrm{R}$ regeneration $(2 \mathrm{R}=\mathrm{Re}$ amplification and Reshaping) in the third part.

The fourth and last part gives an account of some system experiments using both heterogeneously integrated lasers and heterogeneously integrated detectors. The interconnect links that are proposed in this part can be completely fabricated in a CMOS pilot line.

\section{DESIGN AND FABRICATION}

Optimised designs of microdisk lasers are aimed towards minimizing the cavity losses. The internal loss consists of bend losses, scattering loss due to surface wall roughness, intervalence band absorption (IVBA) in p-doped contact layers and losses due to overlap of the whispering gallery mode with the top metal contact. Scattering loss is minimized by utilizing optimized inductively coupled plasma (ICP) etching, while IVBA has so far been limited by the incorporation of a tunnel junction in the epitaxial structure to avoid heavily p-doped regions.

Bend losses decrease with increasing disk radius and depend also on the thickness of the bottom contact layer. However, at least for disk radii beyond $3 \mu \mathrm{m}$, the loss is dominated by other factors [19]. Microdisk lasers with diameters beyond $10 \mu \mathrm{m}$ (e.g., 20 and $40 \mu \mathrm{m}$ ) have so far not given higher output powers than those with diameters of 7.5 and $10 \mu \mathrm{m}$, but they have higher threshold current and can be multi mode. The threshold current density was generally found to be fairly constant for disk diameters above $8 \mu \mathrm{m}$, but increase for smaller diameters due to increased bend and scattering losses [20].
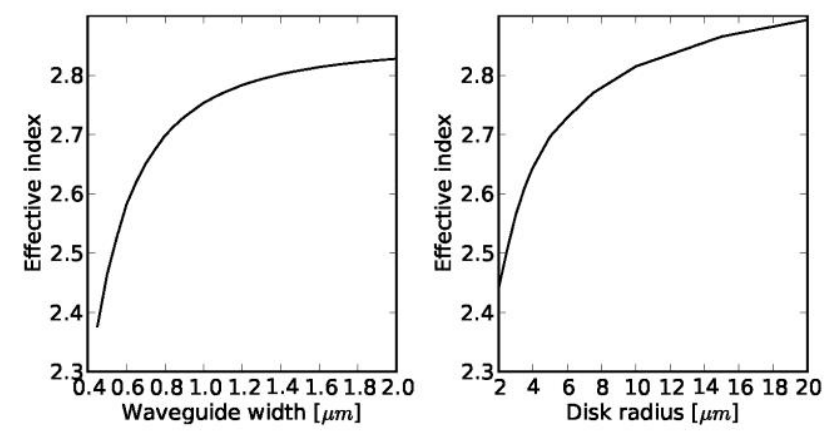

Figure 2. (a) Effective index of the fundamental mode in a $220 \mathrm{~nm}$ thick silicon waveguide vs. waveguide width, (b) Effective index for azimuthal propagation of the fundamental mode in a $580 \mathrm{~nm}$ thick microdisk vs. disk radius.

To avoid large threshold currents, the coupling loss (or facet loss) should not be much larger than the internal loss, while it should also not be much smaller than the internal loss to guarantee a sufficient external efficiency.

The coupling between the microdisk's whispering gallery mode and the underlying silicon waveguide relies on evanescent coupling and depends on the thickness of the bonding layer, the width of the silicon waveguide and the alignment between silicon waveguide and microdisk. The width of the Si waveguide is chosen so as to obtain phase matching with the microdisk mode. Figure 2 shows simulated values for the effective index of the fundamental mode of both a $220 \mathrm{~nm} \mathrm{Si}$ waveguide with variable width and a $580 \mathrm{~nm}$ thick microdisk with variable radius $\mathrm{R}$ [20]. The effective index considered for the microdisk mode is that for the (azimuthal) propagation along the disk perimeter. The values were obtained assuming that the maximum mode intensity is obtained at the disk radius $\mathrm{R}$ and they are therefore lower limits. If a microdisk radius of $3.5 \mu \mathrm{m}$ is considered, a Si waveguide width of slightly over $600 \mathrm{~nm}$ gives the best phase matching.

The alignment of the disk with respect to the Si waveguide must be chosen so as to maximize the coupling between the fundamental disk mode and the fundamental waveguide mode and to minimize the coupling between the fundamental disk mode and higher order (leaky) waveguide modes (and vice versa). Simulation results indicate that a waveguide width of $800 \mathrm{~nm}$ is even better for this purpose and that the optimum offset of $300 \mathrm{~nm}$ away from the waveguide middle gives the least parasitic coupling.

The bonding of the InP dies to the SOI dies can be done using direct [21], molecular (silica to silica) bonding [22] and adhesive bonding [23]. Direct and molecular bonding allow much better heat dissipation than adhesive bonding, as the thermal conductivity of the adhesive used for bonding is rather poor. However, they also require a much better wafer quality (in terms of roughness, defects,...). On the other hand, adhesive bonding layers can be widely varied in thickness, from $20 \mathrm{~nm}$ to $2 \mu \mathrm{m}$.

In the case of adhesive bonding, DVS-BCB (divinylsiloxane -benzocyclobutene) is used as adhesive, which is also used to planarize the SOI die. DVS-BCB reportedly has marginal adhesion to III-V semiconductors, so it was decided to employ an intermediate silica (or alumina) layer deposited on the InP prior to bonding. Moreover, using a controllable thickness for the silica (or alumina) and a very thin DVSBCB layer (e.g. 30nm) allows much better control of the total bonding layer thickness since only the relative $\mathrm{BCB}$ layer thickness can be controlled well. Silica (and even more alumina) are also better thermal conductors than DVS-BCB. Bonding with a rather thick oxide layer and a thin DVSBCB layer thus gives improved heat sinking [24]. To improve the uniformity and reproducibility of the bonding layer, an optimum solution of DVS-BCB:mesitylene has been sought and with the proportions 2:3 a uniform bonding 
layer was obtained, without any air trapping for a SOI die with air clad waveguides [24].

Machine bonding instead of manual bonding gave further improvements in the bonding yield, uniformity and reproducibility [25]. In this case, very thin bonding layers of $35 \mathrm{~nm}$ can be obtained using a DVS-BCB:mesitylene dilution of 1:8 (v/v) and spin-coating at $3000 \mathrm{rpm}$. Bonding yields are now well over $90 \%$ and close to $100 \%$. In the case of machine bonding, the $\mathrm{BCB}$ is precured before bonding on a hot plate for 15 minutes at $180{ }^{\circ} \mathrm{C}$. The bonded samples are for both machine and manual bonding further cured under an $\mathrm{N}_{2}$ flow, with temperatures slowly being increased to $280{ }^{\circ} \mathrm{C}$. The dies are kept at this temperature for one hour and are then slowly cooled down to room temperature. Figure 3 is a SEM image of a sample where $100 \mathrm{~nm}$ of $\mathrm{SiO}_{2}$ has been deposited on the III-V prior to bonding and for which the DVS-BCB bonding layer is $120 \mathrm{~nm}$ (obtained with the $2: 3$ dilution).

The small volume of the microdisk lasers and the bad thermal conductivity of the DVS-BCB and the buried oxide imply a rather bad heat sinking for the laser. The thermal roll-over of microdisk lasers is therefore typically occurring at currents of a few $\mathrm{mA}$. Attention has therefore been paid to a reduction of the heating as well as to an improvement of the heat sinking. The latter can be obtained among others by using a somewhat thicker bottom contact InP layer and an overcladding with oxide instead of BCB. We also employed a thick gold layer, which serves as heat sink, as part of the top contact. Finally, addition of diamond nanoparticles to the $\mathrm{BCB}$ bonding adhesive has proven to improve the heat flow as well [26].

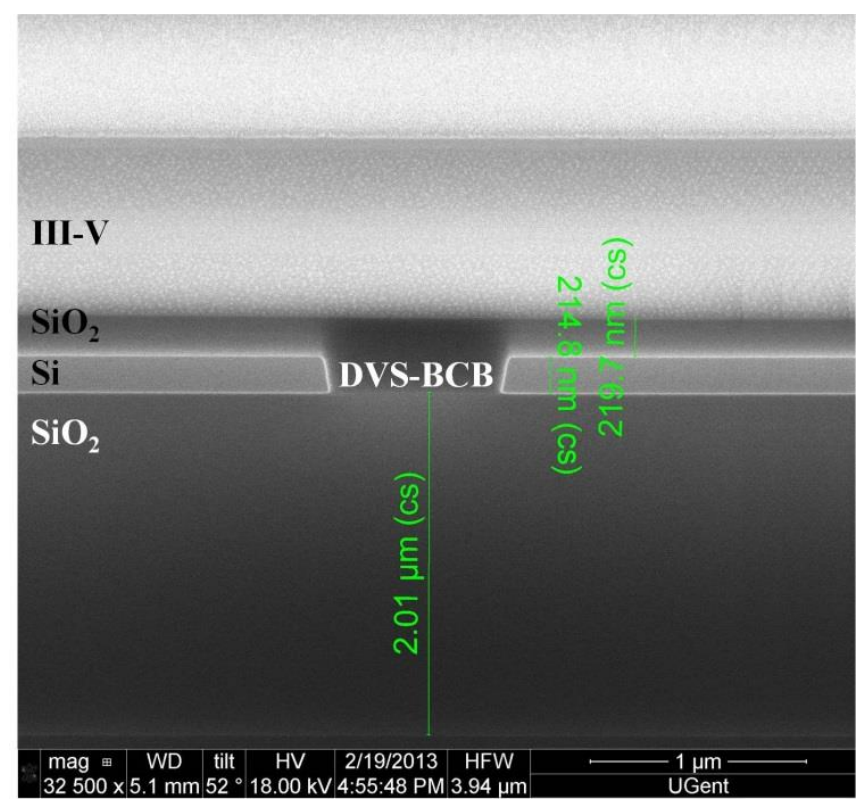

Figure 3. FIB cross-section of a device where a 100nm thick $\mathrm{SiO} 2$ layer has been deposited prior to bonding with the wafer bonder. The measured total bonding thickness is $220 \mathrm{~nm}$ and the DVS-BCB layer thickness is $120 \mathrm{~nm}$.

A reduction of the heating itself requires reducing the optical loss and improving the efficiency of the laser. This requires optimum alignment of the top contact to avoid any overlap of the whispering gallery mode with the top contact to lower optical losses and optimum alignment of the microdisk laser with the underlying silicon wire to ensure an efficient coupling.

Increasing the bottom contact InP layer thickness from 90 to $130 \mathrm{~nm}$ also substantially decreased the series resistance. Optimisation of the bottom contact layer thickness and of the contact recipe led to a decrease of the series resistance from $1 \mathrm{kOhm}$ to $150 \mathrm{Ohm}$. The confinement of the whispering gallery mode was practically unaffected by an increase in bottom contact layer thickness.

E-beam lithography recipes have been developed to optimize the alignment of both the midrodisk with respect to the underlying waveguide and the top contact with respect to the microdisk [27]. Markers for the alignment of the microdisk with respect to the $\mathrm{Si}$ waveguide are defined on the SOI with a resolution of $20 \mathrm{~nm}$. The microdisk and the top contacts were defined with a $2.5 \mathrm{~nm}$ resolution. The accuracy of the alignment of the microdisk lasers with respect to the silicon waveguides was found to be better than $40 \mathrm{~nm}$.

In addition to improved top contact alignment and improved disk vs. Si waveguide alignment, use of this lithography also resulted in a much better uniformity of the dimensions and characteristics of microdisk lasers. In [18], a standard deviation in lasing wavelength of nominally identical devices on the same SOI die lower than 500pm has been reported. The deviation in the diameter of the microdisks as low as a few nanometers makes all-optical signal processing applications requiring cascadability possible.

Figure 4 illustrates the uniformity in lasing wavelength characteristics obtained from 43 microdisk lasers fabricated on a same SOI die. This variation is small enough to allow aligning all laser wavelengths with just a few $\mathrm{mW}$ of thermal tuning. Such thermal tuning can be implemented using InP heaters configured as rings around the microdisks [3].

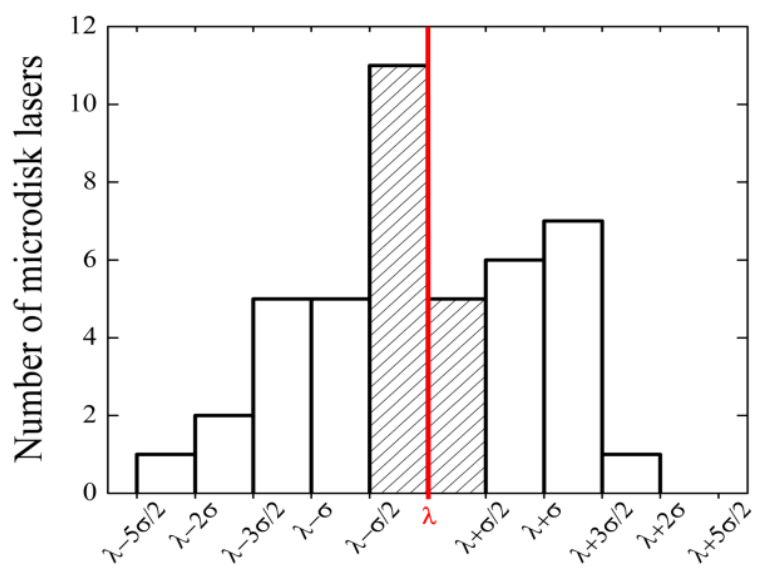

Figure 4. Wavelength distribution of 43 microdisk lasers from the same sample centered on $\lambda$. The patterned area shows that the peak lasing wavelength of 16 microdisk lasers is between $\lambda-\sigma / 2$ and $\lambda+\sigma / 2$ (with $\sigma=500 \mathrm{pm}$ ).

An optimum alignment of the top contact is also possible without the use of e-beam lithography, by using a ring shaped nitride hard mask instead of a disk shaped hard mask. Figure 5 illustrates how this hard mask leads to a self- 
aligned top contact even with a misalignment in the metal deposition. [8]

A last issue concerns the epitaxial III-V layer structure. So far, almost all fabricated microdisk lasers made use of a III$\mathrm{V}$ epitaxial structure with a tunnel junction. This tunnel junction (consisting of two 20nm thick, heavily doped Q1.2 InGaAsP layers) was at first considered necessary to avoid the strong absorption in the $\mathrm{p}++\mathrm{InGaAs}$ contact layer. The InP membrane is only about $600 \mathrm{~nm}$ thick and the mode profile of the whispering gallery mode extends to the top of the InP structure.

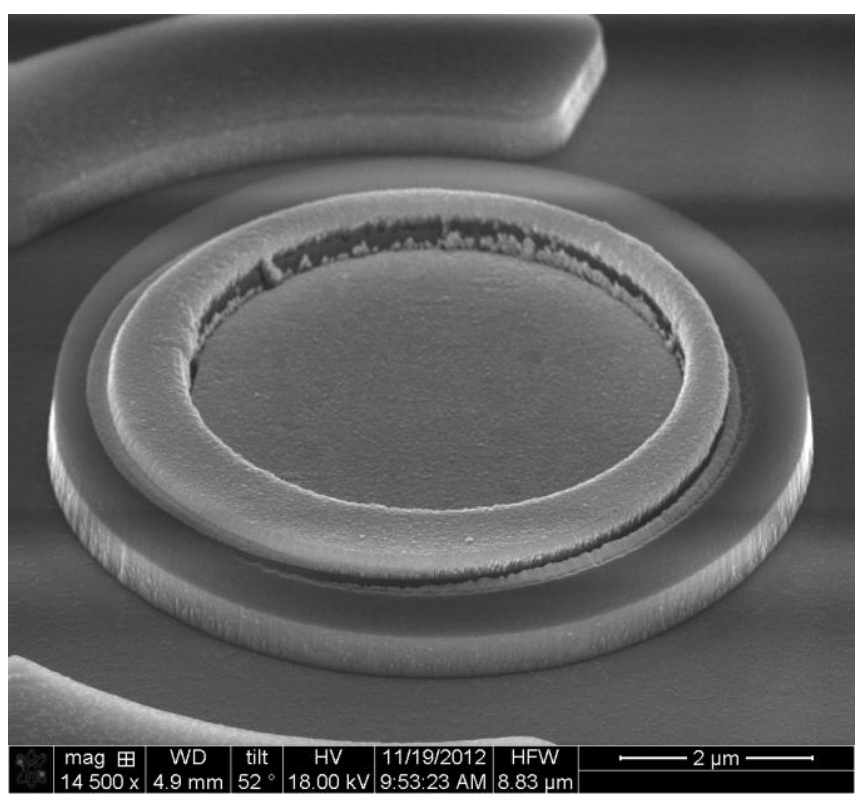

Figure 5. SEM image of an etched microdisk with a self aligned top contact. It can be seen that the Titanium-gold metal itself is misaligned, but the metal area that contacts the disk is perfectly in the center thanks to the nitride ring.

Recently, some studies were carried out with a normal p-i-n layer stack, in which the top p++ InGaAs contact layer is etched away at the edges of the disk to avoid overlap of the whispering gallery mode with this absorbing layer. This is illustrated in Figure 6, where the cross section of the microdisk is shown with the regular tunnel junction containing epitaxial structure as well as with a regular pin junction. Etching the highly absorbing contact layer at the edges results in a whispering gallery mode that is still confined to the membrane and that does not overlap with the highly absorbing p++ InGaAs contact layer. Since the tunnel junction itself also causes significant losses for the mode, the pin structure is expected to result in lower loss for the lasing mode. Numerical simulations confirm the lower modal loss and indicate that the carrier injection efficiency into the active layer is similar as in the structure with tunnel junction.

Figure 7 shows the calculated whispering gallery mode for the original InP structure with thin bottom contact layer (a) and for the same InP structure with thicker bottom contact layer and etched to below the tunnel junction at the edge (b). Both increasing the thickness of the bottom contact layer and etching away the edge in the top contact and tunnel junction layers have little effect on the mode confinement, the bend loss and on the overlap of the mode with the quantum wells. The microdisk diameter was taken as $7.5 \mu \mathrm{m}$. The active layer used in these simulations and in the fabricated devices consists of an SCH-MQW structure, consisting of $36 \mathrm{~nm}$ thick InAsP wells, $315 \mathrm{~nm}$ thick Q1.2 InGaAsP barriers and $25 \mathrm{~nm}$ Q1.2 InGaAsP separate confinement layers. The InP wafers were grown by molecular beam epitaxy at INL, France [28]. InAsP quantum wells are believed to show lower surface recombination than InGaAsP quantum wells [29].

(a)

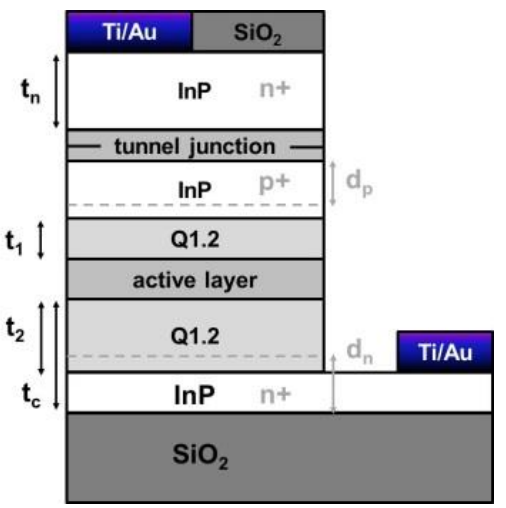

(b)

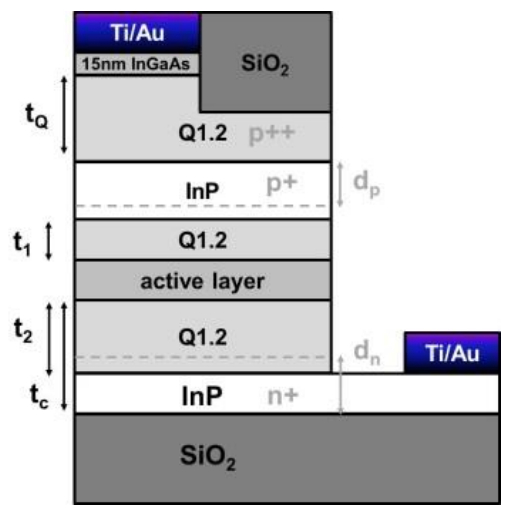

Figure 6. Schematic structure of the InP microdisk structure for two alternative epitaxial layer stacks: (a) with the epilayer structure including a tunnel junction, (b) layer structure with a regular pin junction, of which the highly absorbing contact layers are etched away at the edge.

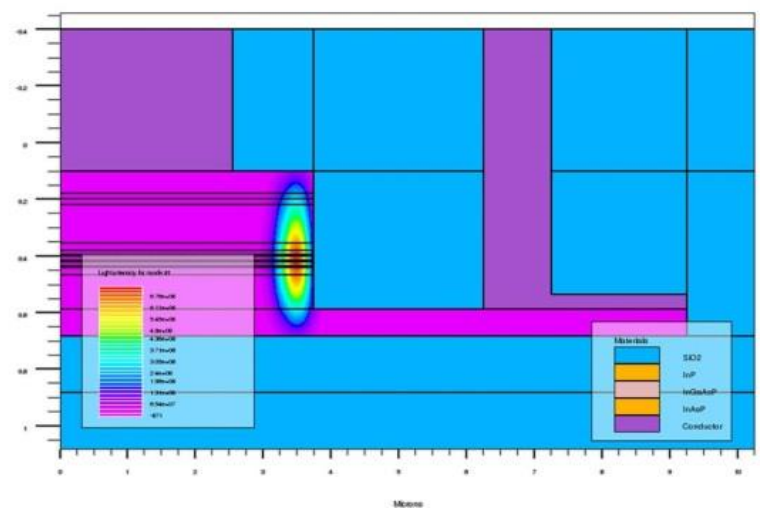

(a) 


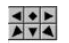

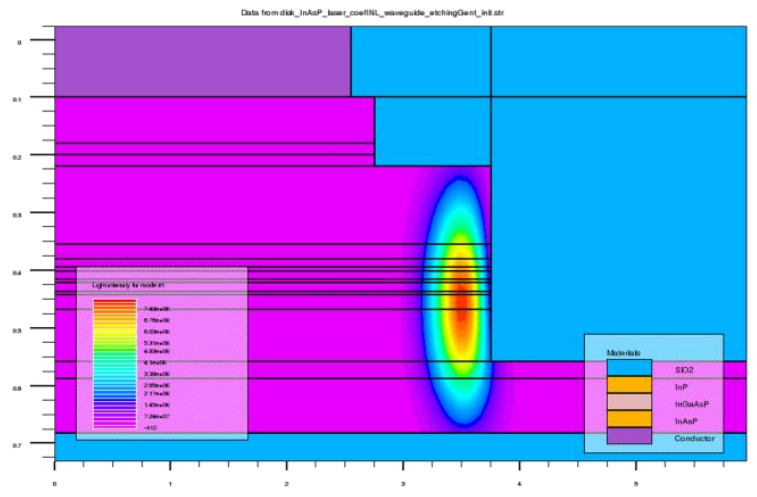

(b)

Figure 7. Fundamental mode profile of the whispering gallery mode in the microdisk for an InP structure with (a) thin bottom contact layer and unetched top layers and (b) thicker bottom contact layer and etched top layers.

\section{UNIDIRECTIONALITY AND REFLECTION SENSITIVITY}

To keep both cost and complexity low, efficient and isolator-free operation are of great importance for light sources for optical interconnects. Bistable unidirectional operation has been studied intensively for ring and disk lasers $[7,30]$ and can potentially be used in optical logic. In optical interconnect though, monostable unidirectional operation is required to maximize the external efficiency of the transmitters.

Such unidirectional behaviour has been reported before $[10,11]$ and has recently been analysed theoretically [9]. Unidirectional behaviour is easily obtained if the coupling between the $\mathrm{CW}$ and the $\mathrm{CCW}$ mode is different from the coupling between the $\mathrm{CCW}$ and $\mathrm{CW}$ mode, e.g., if the microdisk laser is coupled to a bus waveguide which has strong reflection on one side and weak reflection at the other side, as in Figure 8. Lasing is then mainly in the direction which encounters the smallest reflection. For ease of reading and notation, we will assume that this is the $\mathrm{CW}$ direction.

In [11], unidirectional operation was observed in microdisk lasers with $7.5 \mu \mathrm{m}$ diameter, coupled to a silicon bus waveguide that had a strong Bragg reflector (50 periods of $300 \mathrm{~nm}$ each) on one side, designed at $55 \mu \mathrm{m}$ from the microdisk-bus coupler. Output power on both sides was measured vs. bias current and vs. wavelength and the result is shown in Figure 9 for a particular microdisk laser. At the peak lasing wavelength, the extinction ratio of the optical powers coupled out of the waveguide on the side of the DBR structure and on the side without the DBR is $46.1 \mathrm{~dB}$. The transmission of the DBRs was derived from measurements on nominally identical SOI designs, covered in DVS-BCB and was found to be $-38 \mathrm{~dB}$. The $46.1 \mathrm{~dB}$ extinction found on the spectrum thus corresponds with a ratio between $\mathrm{CW}$ and $\mathrm{CCW}$ powers in the disk of $8 \mathrm{~dB}$. Moreover, in Figure 9 (top) there is a clear lasing threshold visible just above $0.5 \mathrm{~mA}$ for the $\mathrm{CW}$ curve, but not for the CCW curve.

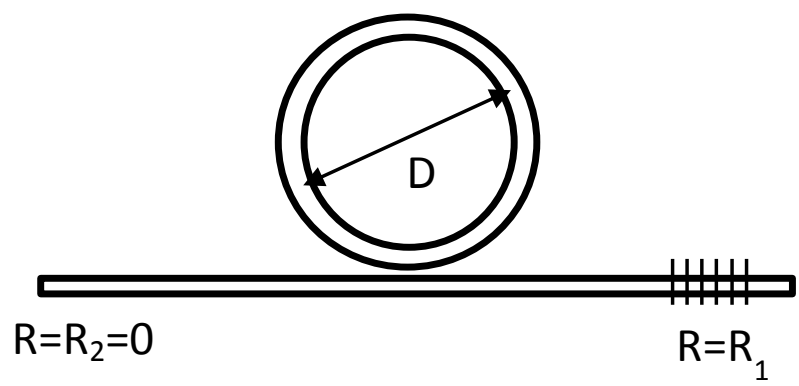

Figure 8. Schematic structure of a ring/disk laser coupled to a bus waveguide, containing a reflector on one side.
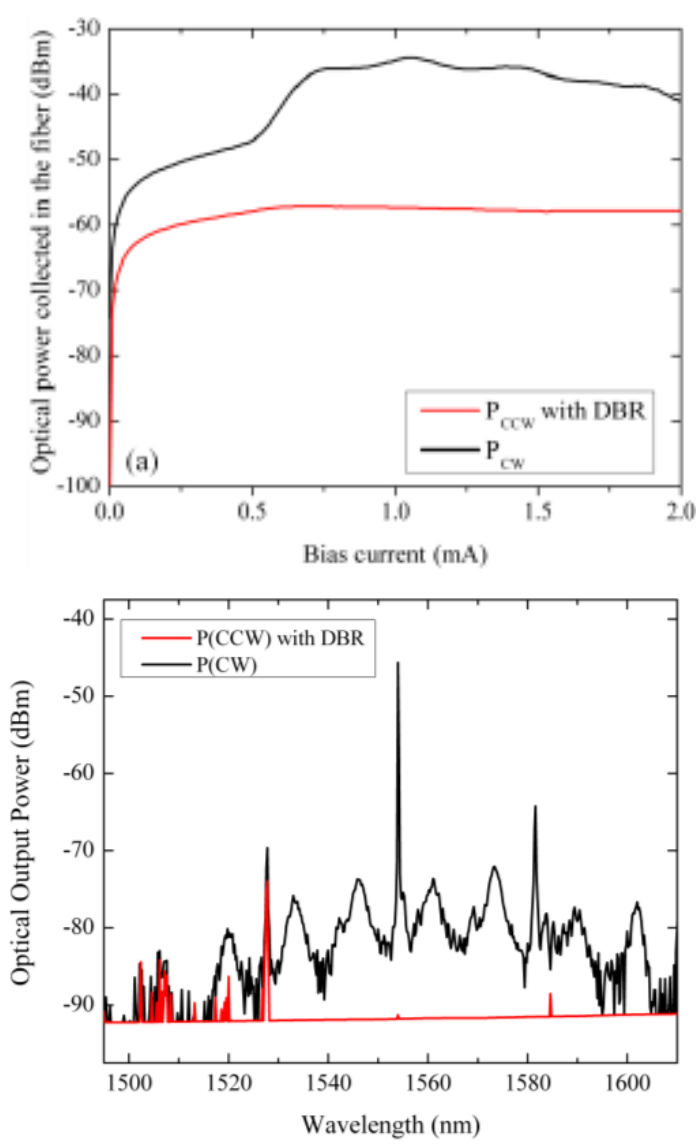

Figure 9. L-I characteristics (top) and spectrum at $1.2 \mathrm{~mA}$ (bottom) of a microdisk laser coupled to a waveguide with a Bragg grating on one side. The optical power is the power collected in optical fibers on both sides of the waveguide.

It has been shown in [9] that the degree of unidirectionality (the ratio of the powers in the two directions $\mathrm{P}_{\mathrm{CW}} / \mathrm{P}_{\mathrm{CCW}}$ ) at low bias (power) levels is simply given by the ratio of the coupling coefficients $\left|K_{1} / K_{2}\right|$ with $K_{1}$ and $K_{2}$ the coupling from $\mathrm{CCW}$ to $\mathrm{CW}$ and vice versa. These coupling coefficients take into account both distributed coupling (e.g. due to scattering at sidewall surface roughness) and coupling due to discrete reflections (at Bragg gratings, facets,...). It has been shown in [7] that information about the coupling coefficients can be derived from the relative intensity noise (RIN) spectra of the microdisk laser.

At high power levels however, unidirectional operation is significantly enhanced by the gain suppression or, more exact, the fact that the cross gain suppression is twice the 
self gain suppression. The ratio of the powers $\mathrm{P}_{\mathrm{CW}} / \mathrm{P}_{\mathrm{CW}}$ then approaches:

$\frac{P_{C W}}{P_{C C W}} \approx \frac{\varepsilon \mathrm{G}_{0} \mathrm{P}_{\mathrm{CW}}}{2\left|K_{2}\right|}$

with $\mathrm{G}_{0}$ the threshold gain (per unit time), $\varepsilon \mathrm{P}_{\mathrm{CW}}$ the self gain suppression for the dominant mode and $\mathrm{K}_{2}$ the smallest of the coupling coefficients (also per unit time).

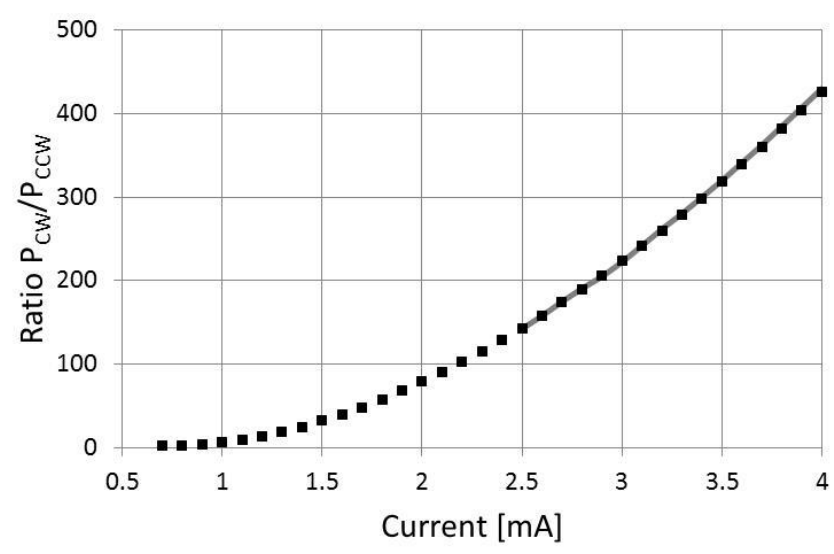

Figure 10. Ratio of power in $\mathrm{CW}$ and $\mathrm{CCW}$ modes, obtained from a numerical solution of the coupled rate equations, for a microdisk laser with coupling coefficients $\mathrm{K}_{1}=6.3610^{8} \mathrm{~s}^{-1}, \mathrm{~K}_{2}=3.1810^{8} \mathrm{~s}^{-1}$, and $(-)$ obtained from expression (23). $\varepsilon=10^{-18} \mathrm{~cm}^{3}$.

Figure 10 shows $\mathrm{P}_{\mathrm{CW}} / \mathrm{P}_{\mathrm{CCW}}$ obtained from a time domain numerical solution of the coupled wave equations up to higher current levels for the case, $\mathrm{K}_{1}=6.3610^{8} \mathrm{~s}^{-1}, \mathrm{~K}_{2}=3.18$ $10^{8} \mathrm{~s}^{-1}$, and $\varepsilon=10^{-18} \mathrm{~cm}^{3}$ as well as the results for currents above $2.5 \mathrm{~mA}$ obtained using expression (1). Although the ratio $\mathrm{K}_{1} / \mathrm{K}_{2}$ is only 2 , one obtains much higher $\mathrm{P}_{\mathrm{CW}} / \mathrm{P}_{\mathrm{CCW}}$ values and they correspond quite well to the values obtaind using (1).

The unidirectional behaviour can also lead to a lower sensitivity to external reflections, especially in the high power regime. If a microdisk laser (or ring laser) is lasing in a unidirectional mode, external reflections don't couple back or couple only weakly into the lasing mode. The ideal case is having no scattering within the disk and the bus waveguide having a perfectly AR-coated facet on one side and a low reflection at the other side.

We derive the feedback sensitivity from the change in angular frequency $\Delta \omega$ caused by the external reflection $[31,32]$. For edge-emitting and vertical cavity surface emitting lasers, $\Delta \omega$ is traditionally expressed as [9]:

$\Delta \omega=\mathrm{C} \sqrt{1+\alpha^{2}} \sin \left[\omega \tau_{e}+\operatorname{arctg}(\alpha)\right] r_{e}$

With $r_{e}$ the external field reflection, $C$ the feedback sensitivity, $\alpha$ the linewidth enhancement factor and $\tau_{\mathrm{e}}$ the delay time of the external reflection.

The feedback sensitivity can always be reduced by increasing the laser facet reflectivities. This is however at the expense of the external efficiency $\eta$ and it has even been shown that the feedback sensitivity $\mathrm{C}$ and the mirror loss $\alpha_{\text {end }}$ are proportional [31]. For a Fabry-Perot laser with one $100 \%$ reflecting facet at the r.h.s. and a field reflection $r_{1}$ at the other (1.h.s.) facet, one can write:

$$
\begin{aligned}
& |\mathrm{C}|=\frac{2}{\tau_{\mathrm{L}}} \alpha_{\mathrm{end}} \mathrm{L} \sqrt{\mathrm{K}_{\mathrm{z}}} \\
& \frac{|\mathrm{C}| \tau_{\mathrm{L}}}{\alpha_{\mathrm{end}} \mathrm{L}}=\frac{1-\left(\mathrm{r}_{1}\right)^{2}}{-\mathrm{r}_{1} \ln \left(\mathrm{r}_{1}\right)}
\end{aligned}
$$

With $\mathrm{K}_{\mathrm{z}}$ the longitudinal Petermann factor and $\tau_{\mathrm{L}}$ the roundtrip time in the Fabry-Perot laser cavity.

For an ideal microdisk laser without scattering inside the disk, a coupling $\kappa$ between disk and bus waveguide and a field reflection $r_{1}$ at one facet and an undesired external reflection $r_{e}$ at the other facet one can express $\Delta \omega$ as [9]:

$$
\begin{aligned}
& \Delta \omega=r_{e}|\mathrm{C}| \sqrt{1+\alpha^{2}} \sin \left(\omega \tau_{\mathrm{e}}+\arg \left(\mathrm{r}_{1}\right)-\tan ^{-1} \alpha\right) \\
& \frac{|\mathrm{C}| \tau_{\mathrm{L}}}{\alpha_{\mathrm{end}} \mathrm{L}}=\frac{2 \kappa^{4}}{-\ln \left(1-\kappa^{2}\right) \tau_{\mathrm{L}}} \frac{r_{1}}{\varepsilon G_{0} P_{C W}}
\end{aligned}
$$

With $r_{1}$ the field reflection of the r.h.s. facet (or of the Bragg grating as in Figure 8). $r_{1}$ can be chosen relatively small since it must just be larger than $r_{e}$ to guarantee unidirectional operation at high output powers.

Since $\tau_{\mathrm{L}}$ is typically of the order of ps or sub-ps, and $\varepsilon G_{0} P_{C C W}$ is of the order of $10^{10} \mathrm{~s}^{-1}$, the last factor in eq. (4) is 100 or more. For values of $\kappa^{2}$ smaller than $1 \%$ and $r_{e}$ smaller than 0.1 , the normalized value of $\mathrm{C}$ can be smaller than that of an equivalent Fabry-Perot laser (with facet reflectivity R1 larger than 99\%). In both cases, the low feedback sensitivity is at the expense of the efficiency. The feedback sensitivity of microdisk lasers improves further with increasing power and decreasing $r_{1}$ though. The reflection sensitivity in this regime strongly depends on the gain suppression value. In our simulations and analytical approximations, we have taken a modest value for $\varepsilon=10^{-18} \mathrm{~cm}^{3}$, but much higher values have been reported in literature $[33,34]$, which give a much lower reflection sensitivity.

In general, with very low sidewall surface roughness, relatively high gain suppression values, low disk-bus coupling and if sufficiently high output powers can be reached, parasitic reflections should not pose a problem for optical interconnects using microdisk lasers.

\section{LOW POWER OPTICAL 2R REGENERATION}

Ring and disk lasers have so far been used in the demonstration of a variety of nonlinear all-optical functions. This includes all-optical flip-flops [10], optical gates and wavelength converters, etc. Typical bitrates that can be handled are 10 to $20 \mathrm{~Gb} / \mathrm{s}$ [3] and switching occurs with sub-pJ-pulses (1.8fJ for AOFF, 160fJ for gating) in times of 50 to $100 \mathrm{ps}$. All these optical switching functionalities require the input signal wavelength to be within the injection locking bandwidth of the laser or within the resonance bandwidth of the resonator (in case the microdisk is biased below threshold). In principle, the lasing or resonance 
wavelength of microdisk lasers can be thermally tuned with relatively lower tuning power [3] and the injection locking requirement is not a major drawback.

Signal restoration or regeneration has been listed before (a.o. in [35]) as one of the key optical functionalities which must be possible in optical logic circuits. Regnerators show great promise for future large scale interconnect networks if the required optical input power and the device power consumption is lowered.

However, of the many all-optical regeneration schemes that have been reported in the past $[36,37]$, none were able to operate with very low input powers and with low power consumption.

We have recently presented an all-optical regeneration scheme based on asymmetric microdisk lasers that could operate with sub-mW input powers and with less than $10 \mathrm{~mW}$ of electrical power dissipation [38]. We will explain the scheme using Figure 8 , assuming $\mathrm{R}_{1}$ to be larger than $\mathrm{R}_{2}$, and thus $\left|K_{1} / K_{2}\right|>1$. The optical beam to be regenerated is injected from the low reflection side, i.e. from the left hand side. The regeneration scheme itself relies on the fact that an external signal within the injection-locking bandwidth of the laser, injected at the low reflection (or left hand) side, is equivalent to a reflection for the laser field at that side. If no or little light is injected from the left hand side, the equivalent $\left|\mathrm{K}_{1} / \mathrm{K}_{2}\right|$ will remain larger than one. The microdisk (or ring) laser will operate in the $\mathrm{CW}$ direction and little or no light will be coupled out from the right hand side. Injection of sufficiently high power at the left hand side (into the $\mathrm{CCW}$ direction) however will cause an equivalent reflection at the left hand side, which is larger than that at the right hand side and it will cause the equivalent $\left|\mathrm{K}_{1} / \mathrm{K}_{2}\right|$ to become smaller than one. The lasing direction will therefore switch from the $\mathrm{CW}$ to the $\mathrm{CCW}$ direction and a relatively high power will be coupled out from the right hand side. The threshold power for this switching obviously depends on the asymmetry of the microdisk laser and can be low for a small asymmetry. In this case, a sufficiently high bias will still give a good unidirectional behaviour, as discussed in part III.

Numerical simulations using a simple system of coupled rate equations show this threshold mechanism clearly. Figure 11 shows the output power from the 1.h.s. (CW) and r.h.s. (CCW) facets respectively vs. the input power injected at the 1.h.s. facet. A distinct threshold and a relatively flat output power, ideal for regeneration, are obtained in the $\mathrm{CCW}$ mode. Dynamic simulations at $10 \mathrm{~Gb} / \mathrm{s}$ have furthermore shown the possibility of extinction ratio improvement and noise reduction [38].

Experimentally, regeneration has been demonstrated for a $10 \mathrm{~Gb} / \mathrm{s}$ NRZ signal (PRBS $2^{31}-1$ ), which was attenuated and sent through an EDFA to degrade its signal-to-noise ratio. The measured BER before and after regeneration is given in Figure 12 as a function of the received power. The microdisk laser was biased at $4 \mathrm{~mA}$ and the experiments were carried out with relatively high extinction ratio $(13 \mathrm{~dB})$ for the input signal. Although the regeneration resulted in an output extinction ratio of $16 \mathrm{~dB}$, Figure 11 seems to indicate that even more extinction ratio and BER improvement can be obtained for lower input extinction ratio.

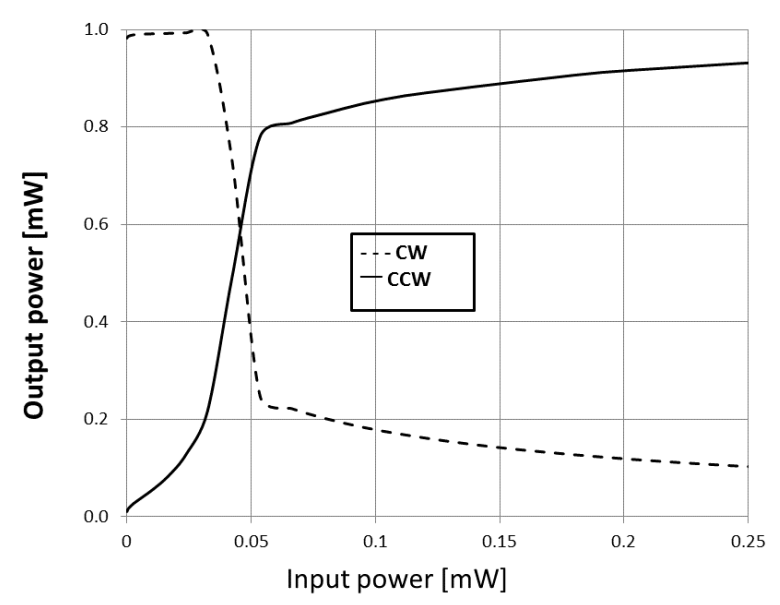

Figure 11. Static regeneration characteristic for a microdisk laser with $\mathrm{K}_{2} / \mathrm{K}_{1}=10$.

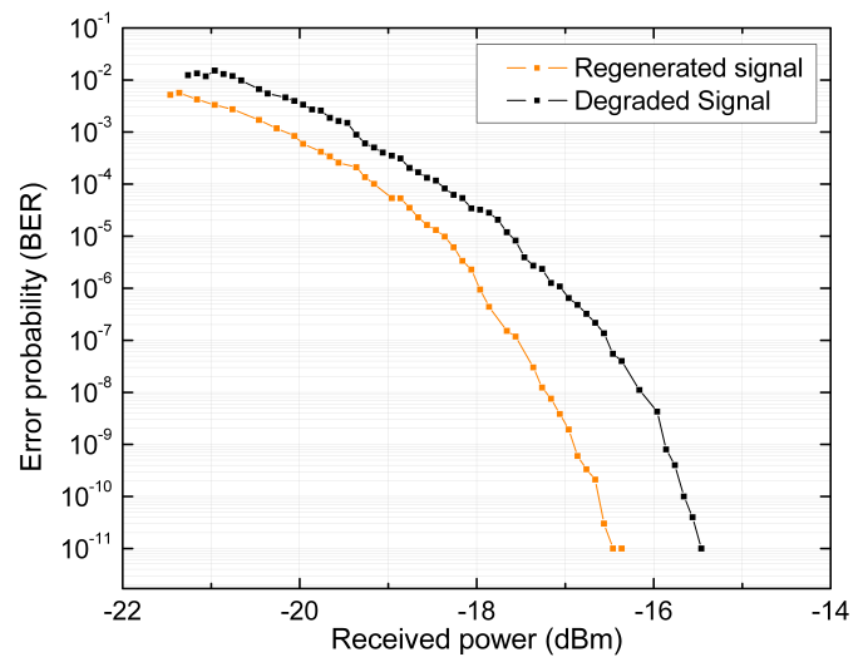

Figure 12. Measured BER curves at $10 \mathrm{~Gb} / \mathrm{s}$, before and after regeneration with a microdisk laser.

\section{OPTICAL INTERCONNECT DEMONSTRATIONS}

When using a heterogeneous integration approach, an obvious route towards optical interconnect on SOI is to use silicon waveguides and the same III-V layer stack for transmitter and detector [40]. However, MQWs are often preferred for the active layer of the laser and the small overlap of the optical mode with the quantum wells make this layer stack less suitable for the implementation of compact detectors. Another possibility is to use separate III$\mathrm{V}$ dies for laser and detector, but they need to be spatially separated, which limits the integration density.

We proposed to use an epitaxial layer stack that contains both the laser and the detector epitaxy, with the detector layers stacked on top of the laser structure. This allows cointegrating detectors with lasers, as shown in Figure 13. The detector epitaxial structure consists of a $100 \mathrm{~nm}$ n-doped InP layer, a $400 \mathrm{~nm}$ undoped InGaAs layer and a 100nm heavily p-doped InGaAs layer. The transmitter epitaxial structure is similar to the one shown in Figure 6(a). The n-doped InP layer acts both as top contact layer for the microdisk laser and as bottom contact layer for the detector, making the total epitaxial layer stack $1083 \mathrm{~nm}$ thick. 
As is the case for the coupling from microdisk laser to silicon waveguide, the coupling from silicon waveguide to the detector is through evanescent coupling. The bonding layer for optimum detection now needs to be as thin as possible though. On the other hand, the $1 \mu \mathrm{m}$ thick and several $\mu \mathrm{m}$ wide detector structure supports multiple optical modes, making it easier to achieve phase matching between the silicon waveguide and the detector waveguide. Simulations indicate that the coupling is mainly to the higher order modes of the detector waveguide. The silicon waveguide width (changed between 0.5 and $3 \mu \mathrm{m}$ ) did not affect the coupling very much, so $500 \mathrm{~nm}$ wide waveguides were chosen for the fabrication.

Fabricated photodetectors of $40 \mu \mathrm{m}$ and $60 \mu \mathrm{m}$ length gave dark currents (at a reverse bias of $1.5 \mathrm{~V}$ ) of 22 and $67 \mathrm{nA}$. Responsivities were 0.69 and $0.73 \mathrm{~A} / \mathrm{W}$ respectively [41]. The dark current increases significantly with detector length due to the increased sidewall surface area, while the responsivity increase with length is marginal. A bandwidth of 12 and $18 \mathrm{GHz}$ was obtained for 0 and $-1 \mathrm{~V}$ bias.

\section{Waveguide detector}
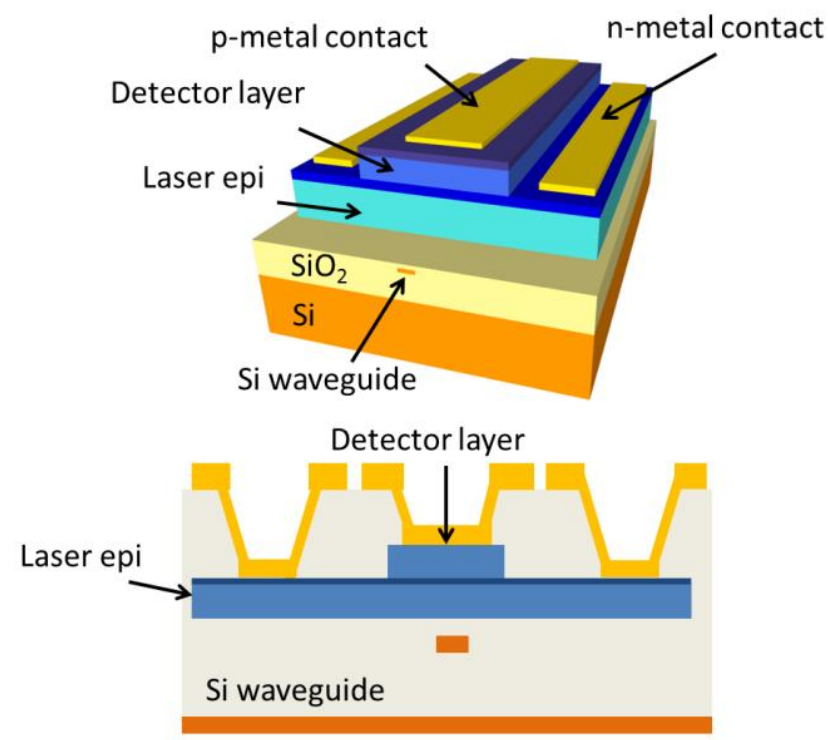

Figure 13. Schematic overview of the detector design, where (a) shows a 3D impression and (b) shows the cross section of the detector.

We have investigated two different link designs: a compact single link design with electrical contacts close to the devices for direct probing and a design containing multiple links, which can be driven by directly flip-chipping a CMOS control chip on top. Figure 14 shows a current-current plot under pulsed driving conditions for the single link. Figure 15 shows the large signal modulation response at $10 \mathrm{~Gb} / \mathrm{s}$ of the link. The direct modulation bandwidth of the microdisk laser was $7.8 \mathrm{GHz}$.

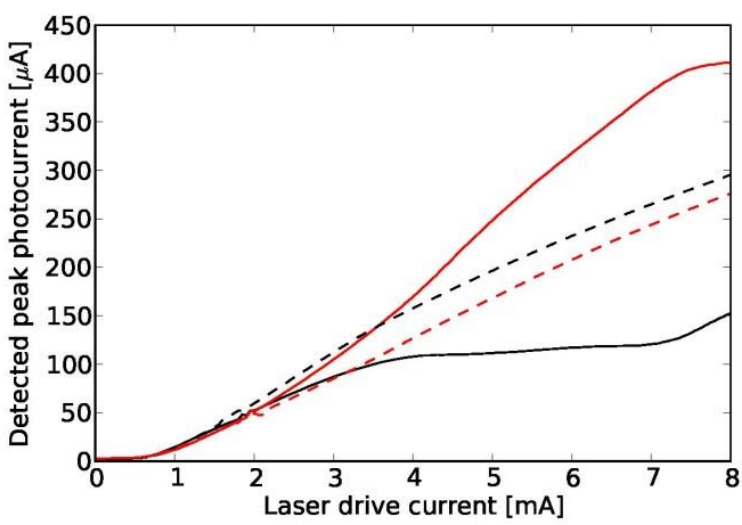

Figure 14. Current versus current plot for two different optical links (dashed and solid lines) under pulsed drive conditions. The black and red curves correspond with the left and right detector, respectively.

As an alternative for the III-V waveguide detector, one could of course also a Germanium detector [42]. Finally, direct modulation of microdisk lasers has been demonstrated at $20 \mathrm{~Gb} / \mathrm{s}$ (using injection locking), with $1 \mathrm{~mW}$ bias power and $190 \mathrm{mV}$ (50 fJ/bit) data signals [43]. The low required drive voltages are compatible with standard CMOS logical levels. As discussed in [3], multi wavelength transmitters can be implemented by fabricating arrays of microdisks with different diameter, all coupled to the same bus waveguide. By providing InP heaters around the microdisks, thermal tuning of the lasing wavelength is possible with an efficiency of $0.3 \mathrm{~nm}$ per $\mathrm{mW}$ of heating power [44].

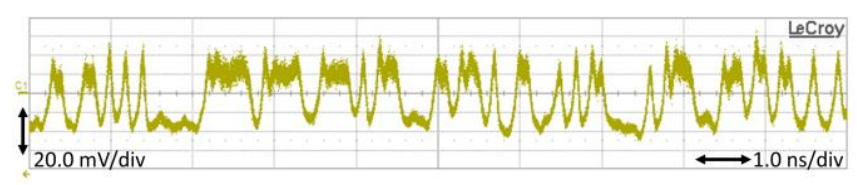

Figure 15. Large signal modulation response of the full optical link for a $10 \mathrm{~Gb} / \mathrm{s} 2^{7}-1$ PRBS pattern.

\section{CONCLUSION}

Recent developments in the fabrication and new applications of heterogeneously integrated microdisk lasers have been discussed. The bonding of the InP on the SOI occurs with high yield and with controllable thickness of the bonding layer(s). Improvements in processing have allowed achieving much better alignment accuracy, for both the disk with respect to the underlying silicon waveguide and for the top metal contact with respect to the center of the disks. Very good uniformity in lasing characteristics has been demonstrated as a result. A number of measures to reduce the losses for the whispering gallery mode and to improve the thermal behavior have been proposed as well.

Furthermore we have detailed how higher-efficiency, unidirectional lasing can be obtained from asymmetric microdisk lasers. At higher bias levels, such lasers can have a much lower external feedback sensitivity than traditional Fabry-Perot, DFB or DBR lasers or VCSELs. Moreover, 
they can be used as all-optical signal regenerators that operate on low input optical powers and with low power consumption.

Finally, optical interconnects on chip have been demonstrated at $10 \mathrm{~Gb} / \mathrm{s}$. An epitaxial layer stack that contains both the laser and the detector structure has been used for this purpose.

Further optimization in design and fabrication of the microdisk lasers could lead to further improvements in threshold current and output power as well as to reduced coupling between clockwise and counterclockwise modes. Such improvements would in turn make these lasers less sensitive to parasitic reflections, give higher modulation bandwidth and reduce electrical power consumption. They can also easily be operated in a predefined unidirectional mode, and several lasers with slightly different diameter and wavelength can be coupled to a single bus waveguide, thus forming a WDM source, Heterogeneously integrated microdisk lasers therefore could well turn out to be the ideal optical transmitters for silicon-based optical interconnects. 


\section{REFERENCES}

[1] P.R. Romeo, J. Van Campenhout, P. Regreny, A. Kazmierczak, C. Seassal, X. Letartre, G. Hollinger, D. Van Thourhout, R. Baets, J.M. Fedeli, L. DiCioccio, 'Heterogeneous integration of electrically driven microdisk based laser sources for optical interconnects and photonic ICs', Opt. Expr., Vol. 14, Is. 9, pp. 3864-3871, 2006.

[2] D. Liang, M. Fiorentino,S. Srinivasan, S. T. Todd, G. Kurczveil, J. E. Bowers, R. G. Beausoleil, 'Optimization of Hybrid Silicon Microring Lasers’, IEEE Photonics Journ., Vol. 3, pp. 580-587, June 2011.

[3] D. Van Thourhout, T. Spuessens, L. Liu, G. Roelkens, R. Kumar, G. Morthier, P. Rojo-Romeo, F. Mandorlo, P. Regreny, O. Raz, L. Grenouillet, 'Nanophotonic devices for optical interconnect', invited paper IEEE Journ. Sel. Topics. QE., Vol. 16, pp. 1363-1375, Sept.Oct. 2010.

[4] C. Gunn, 'CMOS photonics for high-speed interconnects', IEEE Micro, Vol. 26, no. 2, pp. 58-66, Mar./Apr. 2006.

[5] S. Matsuo, T. Sato, K. Takeda, A. Shinya, K. Nozaki, E. Kuramochi, H. Taniyama, M. Notomi, T. Fuji, K. Hasebe, T. Kakitsuka, 'Photonic crystal lasers using wavelength-scale embedded active region', Journ. Appl. Phys. -D, Vol. 47, Is. 2, Art. Nr. 023001, Jan. 2014.

[6] M. Heck, J. Bowers, 'Energy Efficient and Energy Proportional Optical Interconnects for Multi-Core Processors: Driving the Need for On-Chip Sources', IEEE Journ. Sel. Topics Quant. El., Vol. 20, Nr. 4, Art. Nr. 8201012, Jul-Aug. 2014.

[7] G. Morthier, P. Mechet, 'Theoretical analysis of unidirectional operation and reflection sensitivity of semiconductor ring or disk lasers', IEEE Journ. Quant. El., Vol. 49, pp. 1097-1101, Dec. 2013.

[8] D. Liang, S. Srinivasan, D. A. Fattal, M. Fiorentino, Z. Huang, D. T. Spencer, J. E. Bowers, and R. G. Beausoleil, 'Teardrop ReflectorAssisted Unidirectional Hybrid Silicon Microring Lasers', IEEE Photonics Technol. Lett., Vol. 24, No.22, pp. 1988-1990, 2012.

[9] P. Mechet, S. Verstruyft, T. De Vries, T. Spuesens, P. Regreny, D. Van Thourhout, G. Roelkens, G. Morthier, 'Unidirectional III-V microdisk lasers heterogeneously integrated on silicon', Optics Expres, Vol. 21, Issue 16, pp. 19339-19352, Aug. 2013.

[10] L. Liu, R. Kumar, K. Huybrechts, T. Spuesens, G. Roelkens, E.-J. Geluk, T. de Vries, P. Regreny, D. Van Thourhout, R. Baets, and G. Morthier, 'An ultra-small, low-power, all-optical flip-flop memory on a silicon chip’, Nature Photonics 4, pp. 182-187, 2010.

[11] T. Spuesens, P. Regreny, D. Van Thourhout, 'Improved fabrication process for III-V based Optical Interconnects on Silicon', Group IV Photonics, 2013.

[12] G. Mezosi, M.J. Strain, S. Furst, Z. Wang, M. Sorel, 'Unidirectional bistability in AlGaInAs Microring and Microdisk Semiconductor Lasers', IEEE Photon. Technol. Lett., Vol. 21, pp. 88-90, 2009.

[13] W. Wang, G. Yuan, G. Verschaffelt, J. Danckaert, S. Yu, 'Injection Locking and Switching Operations of a Novel Retro-ReflectorCavity-Based Semiconductor Micro-Ring Laser’, IEEE Phot. Techn. Lett., Vol. 20, pp. 1673-1675,2008.

[14] R. Kumar, T. Spuesens, P. Mechet, N. Olivier, J.-M. Fedeli, P. Regreny, G. Roelkens, D. Van Thourhout, G. Morthier, '10 Gbit/s alloptical NRZ-OOK to RZ-OOK format conversion in an ultra-small
III-V-on-silicon microdisk fabricated in a CMOS pilot line', Opt. Expr., vol. 19, pp. 24647-24656, Nov. 2011.

[15] J. Hofrichter, O. Raz, L. Liu, G. Morthier, F. Horst, P. Regreny, T. de Vries, H. Dorren, B. Offrein, 'All-Optical Wavelength Conversion Using Mode Switching in an InP Microdisc Laser', El. Lett., Vol. 47, pp. 927-929, Aug. 2011.

[16] A. Trita, G. Mezosi, M. Sorel, G. Guiliani,'All-Optical Toggle FlipFlop Based on Monolithic Semiconductor Ring Laser', IEEE Phot. Techn. Lett, Vol. 26, No. 1, pp. 96-99, Jan. 2014.

[17] S. Keyvaninia, M. Muneeb, S. Stankovic, R.van Veldhoven, D. Van Thourhout, G. Roelkens, Ultra-thin DVS-BCB adhesive bonding of III-V wafers, dies and multiple dies to a patterned silicon-on-insulator substrate, Optical Materials Express, Vol. 3, Is. 1, pp.35-46, 2013.

[18] P. Mechet, F. Raineri, A. Bazin, Y. Halioua, T. Spuesens, T. Karle,P Regreny, P. Monnier, D. Van Thourhout, I Sagnes, R. Raj, G. Roelkens, G. Morthier, 'Uniformity of the lasing wavelength of heterogeneously integrated InP microdisk lasers on SOI', Opt. Expr., 21(9), pp. 10622-10631, 2013.

[19] J. Van Campenhout, PhD theis, Ghent University, 2007. (Available at http://photonics.intec.ugent.be/publications/phd.asp).

[20] T. Spuesens, PhD thesis, Ghent University, 2014. (Available at http://photonics.intec.ugent.be/publications/phd.asp).

[21] M. Kostrzewa, L. Di Cioccio, M. Zussy, J. C. Roussin, J. M. Fedeli, N. Kernevez, P. Regreny, C. Lagahe-Blanchard, and B. Aspar, "InP dies transferred onto silicon substrate for optical interconnects application," Sensors and Actuators a-Physical, vol. 125, pp. 411 414, Jan 2006.

[22] D. Liang, J. E. Bowers, D. C. Oakley, A. Napoleone, D. C. Chapman, C. L. Chen, P. W. Juodawlkis, and O. Raday, "High-Quality $150 \mathrm{~mm}$ InP-to-Silicon Epitaxial Transfer for Silicon Photonic Integrated Circuits," Electrochemical and Solid State Letters, vol. 12, pp. H101H104, 2009.

[23] G. Roelkens, J. Brouckaert, D. Van Thourhout, R. Baets, R. Notzel, and M. Smit, 'Adhesive bonding of InP/InGaAsP dies to processed silicon-on-insulator wafers using DVS-bis-benzocyclobutene', Journal of the Electrochemical Society, vol. 153, pp. G1015-G1019, 2006.

[24] P. Mechet, PhD thesis, Ghent University, 2014. (Available at http://photonics.intec.ugent.be/publications/phd.asp).

[25] S. Keyvaninia, M. Muneeb, S. Stanković, P. J. Van Veldhoven, D. Van Thourhout, and G. Roelkens, 'Ultra-thin DVS-BCB adhesive bonding of III-V wafers, dies and multiple dies to a patterned siliconon-insulator substrate', Opt. Mat. Expr., Vol. 3, pp. 35-36, 2013.

[26] A. Bazin, Y. Halioua, P. Monnier, F. Bordas, T. Karle, S. Perruchas, T. Gacoin, H. Girard, I. Sagnes, G. Roelkens, R. Raj, F. Raineri., 'Thermal improvement of InP wire photonic crystal laser on silicon by addition of diamond nanoparticles in polymer bonding layer', ECOC proceedings, Italy, 2010.

[27] T. J. Karle, Y. Halioua, F. Raineri, P. Monnier, R. Braive, L. Le Gratiet, G. Beaudoin, I. Sagnes, G. Roelkens, F. van Laere, D. Van Thourhout, and R. Raj, "Heterogeneous integration and precise alignment of InP-based photonic crystal lasers to complementary metal-oxide semiconductor fabricated silicon-on-insulator wire waveguides,” J. Appl. Phys. 107, 063103, 2010. 
[28] http://inl.cnrs.fr/en/research-groups/item/26-heteroepitaxie-etnanostructures

[29] M Yamamoto, N Yamamoto, and J Nakano, MOVPE growth of strained InAsP/InGaAsP quantum-well structures for low-threshold $1.3 \mu \mathrm{m}$ lasers',. IEEE Journal of Quantum Electronics, Vol. 30, pp. 554-561, 1994.

[30] M.T. Hill, H. Dorren, T. De Vries, X. Leijtens, J.H. Den Besten, B. Smallbrugge, Y. Oei, H. Binsma, G. Khoe, M. Smit , A fast lowpower optical memory based on coupled micro-ring lasers', Nature Vol. 432, pp. 206-209, 2004.

[31] O. Nilsson, J. Buus, 'Linewidth and Feedback Sensitivity of Semiconductor Diode Lasers', IEEE Journ. Quant. El., Vol. 26, pp. 2039-2042, Dec. 1990

[32] R. Tkach, A. Chraplyvy, Regimes of Feedback Effects in $1.5 \mu \mathrm{m}$ Distributed Feedback Lasers, IEEE J. Lightwave Technol., Vol. 4, pp. 1655-1661, 1986.

[33] A. Olivier, P. Brosson, J. Benoit, B. Fernier, M. Gailhanou, D. Leclerc, 'Determination of the gain compression coefficient of GaInAsP $1.5 \mu \mathrm{m}$ multiple quantum well lasers by harmonic distortion measurements', Proc. Of OFC'91, p. 125, San Diego, 1991.

[34] J. Bowers, B. Hemenway, A. Gnauck, D. Wilt, 'High-speed constricted-mesa lasers', IEEE Journ. Quant. El., Vol. 22, pp. 833844,1986

[35] D.A.B. Miller, D. A. B. Miller, "Are optical transistors the next logical step?" Nature Photonics 4, 3 - 5 (2010) doi:10.1038/nphoton.2009.240

[36] J. Leuthold, B. Mikkelsen, R. E. Behringer, G. Raybon, C. H. Joyner, and P. A. Besse, 'Novel 3R regenerator based on semiconductor optical amplifier delayed-interference configuration', IEEE Photon. Technol. Lett., vol. 13, no. 8, pp. 860-862, Aug. 2001.

[37] M. Rochette, L. B. Fu, V. Ta'eed, D. J. Moss, and B. J. Eggleton, 2R optical regeneration: An all-optical solution for BER improvement, IEEE J. Sel. Topics Quantum Electron., vol. 12, no. 4 pp. 736-744, Jul./Aug. 2006.

[38] P. Mechet, T. Spuesens, S. Werquin, K. Vandoorne, N. Olivier, J.-M. Fedeli, P. Regreny, D. Van Thourhout, G. Roelkens,G. Morthier, 'All-optical, low-power $2 \mathrm{R}$ regeneration of $10 \mathrm{~Gb} / \mathrm{s}$ NRZ signals using a III-V on SOI microdisk laser', 2014.

[39] B. Li, M. Irfan Memon, G. Mezosi, Z. Wang, M. Sorel, and S. Yu, 'Characterization of all-optical regeneration potential of a bistable semiconductor ring laser', J. Lightwave Technol., vol. 27, no. 19, pp. 4233-4239, Oct. 2009.

[40] G Roelkens, D Van Thourhout, R Baets, R N“otzel, and M Smit, 'Laser emission and photodetection in an InP/InGaAsP layer integrated on and coupled to a Silicon-on-Insulator waveguide circuit' Optics express, 14(18):8154-9,September 2006.

[41] T. Spuesens, F. Mandorlo, P. Rojo-Romeo, P Regreny, N. Olivier, J.M. Fedeli, D. Van Thourhout, 'Compact Integration of Optical Sources and Detectors on SOI for Optical Interconnects Fabricated in a 200 mm CMOS Pilot Line', Journ. Lightw. Techn., Vol. 30, pp. 1764-1770, 2012.

[42] L. Vivien, J. Osmond, J. M. Fedeli, D. Marris-Morini, P. Crozat, J. F. Damlencourt, E. Cassan, Y. Lecunff, and S. Laval, '42 GHz p.i.n
Germanium photodetector integrated in a silicon-on-insulator waveguide', Optics Express, vol. 17, pp. 6252-6257, Apr 2009.

[43] O. Raz, H. Dorren, R. Kumar, G. Morthier, P Regreny, P. RojoRomeo, '50 fJ-per-bit, High Speed, Directly Modulated Light Sources for On-Chip Optical Data Communications', Proc. OFC'2011, 2011.

[44] L. Liu, T. Spuesens, G. Roelkens, D. Van Thourhout, P. Regreny, P. Rojo-Romeo, A thermally tunable microdisk laser built on a IIIV/Silicon-on-insulator heterogeneous integration platform, Photonics Technology Letters, Vol. 22, pp.1270-1272, 2010. 
Geert Morthier (M'93-SM'01) received the degree in electrical engineering and the Ph.D. degree from the University of Gent in 1987, 1991, respectively. Since 1991 he is a member of the permanent staff of IMEC. His main interests are in the modelling and characterisation of optoelectronic components. He has authored or co-authored over 150 papers in the field and holds several patents. He is also one of the two authors of the Handbook of Distributed Feedback Laser (Artech House, 1997). From 1998 to end of 1999 he has been the project manager of the ACTS project ACTUAL dealing with the control of widely tunable laser diodes, from 2001 to 2005 he was project manager of the IST project NEWTON on new widely tunable lasers and from 2008 to 2011 he was project manager of the FP7 project HISTORIC on microdisk lasesrs. In 2001 he was appointed parttime professor at Ghent University, where he teaches courses on optical fiber communication and lasers.

$\mathrm{He}$ serves on the program committee of several international conferences

Thijs Spuesens received (S'08-M'08) was born in Sas van Gent, The Netherlands, in 1982. He received the M.Sc. degree in electrical engineering from Eindhoven University of Technology, Eindhoven, The Netherlands, in 2008. He is currently working toward the Ph.D. degree in photonics in the Department of Information Technology (INTEC), Ghent University, Ghent, Belgium.

In August 2008, he joined the Photonics Research Group, INTEC. He is also involved in the FP7 project WADIMOS. His current research interests include heterogeneous integration of silicon-on-insulator and III-V material, optical interconnects, and microsources.

Pauline Mechet (S'07-M'09) received the B. Eng. Degree in physics engineering, as well as the Engineer Diploma degree and the M.Sc. degree in Optoelectronics from Grenoble I.N.P. PHELMA, Grenoble, France, in 2007 and 2009, respectively. In 2009 she joined the Photonics Research Group of Ghent University - imec, at the Department of Information Technology (INTEC). In 2014, she received the PhD degree in photonics from Ghent University.

Her current research interests include heterogeneous integration of SiliconOn-Insulator and III-V materials and all-optical signal processing based on microdisk lasers.

Ms. Mechet is a Student Member of IEEE Photonics Society.

Gunther Roelkens received the M.Sc. degree in electrical engineering in 2002 and the Ph.D. degree in electrical engineering in April 2007, both from Ghent University, Belgium He currently is a tenure track professor at Ghent University working in the field of heterogeneously integrated silicon photonics waveguide circuits. $\mathrm{He}$ is also an Assistant Professor at Eindhoven University of Technology, the Netherlands.

Dries Van Thourhout (M'98) received the Master's degree in physical engineering and the Ph.D. degree from Ghent University, Ghent, Belgium, in 1995 and 2000, respectively.

From October 2000 to September 2002, he was with Lucent Technologies, Bell Laboratories, Crawford Hill, NJ, where he was engaged in InPInGaAsP monolithically integrated devices. In October 2002, he joined the Department of Information Technology, Ghent University, where he is involved in integrated optoelectronic devices. His current research interests include heterogeneous integration by wafer bonding, intrachip optical interconnect, and wavelengthdivision multiplexing devices. 